\title{
The First Decade of the IERS
}

\section{Ivan I. Mueller}

Department of Civil and Environmental Engineering and Geodetic Science The Ohio State University Columbus, OH 43210-1275 mueller@mps.ohio-state.edu

\begin{abstract}
The International Earth Rotation Service (IERS) was established in 1987 by the International Astronomical Union (IAU) and the International Union of Geodesy and Geophysics (IUGG), and it began operation on 1 January 1988. The primary objectives of the IERS are to serve the astronomical, geodetic and geophysical communities by providing the following:
\end{abstract}

- The International Celestial Reference System (ICRS) and its realization, the International Celestial Reference Frame (ICRF).

- The International Terrestrial Reference System (ITRS) and its realization, the International Terrestrial Reference Frame (ITRF).

- Earth orientation parameters required to study Earth orientation variations and to transform between the ICRF and the ITRF.

- Geophysical data to interpret time/space variations of the ITRF with respect to the ICRF, i.e., of the Earth orientation parameters, and to model such variations.

- Standards, constants and models (i.e., conventions) encouraging international adherence.

This presentation primarily covers the first three IERS functions from the operational point of view.

\section{Background}

\subsection{Benchmarks}

- 1978-80. The IAU and the IUGG establish the MERIT (Monitor Earth Rotation and Intercompare the Techniques of Observation and Analysis) and COTES (Establish and Maintain a New Conventional Terrestrial Reference System) groups to organize observation and analysis campaigns for the purpose of confirming the capabilities of space-based observational techniques, in existence at the time, to determine Earth orientation parameters. George A. Wilkins describes these campaigns during the period 1978-1987 elsewhere in this volume. 
- 1985 August 3, Columbus, OH. The MERIT/COTES Working Group recommends to establish the IERS (see e.g., Bul. Géod. 60, pp. 85-100, 1986).

- 1985 November 22, New Delhi, India. IAU Resolution B2 - Reference Frames endorses the Working Group recommendations and sets up a Provisional Directing Board to submit recommendations for the IERS Terms of Reference.

- 1987 August 17, Vancouver, BC. IUGG Resolution No. 1 endorses the Provisional Directing Board recommendations on the Terms of Reference and decides, in collaboration with the IAU, to establish the IERS on 1988 January 1.

- 1999 March 30. The IERS Directing Board adopts a new Terms of Reference (see http://hpiers.obspm.fr), which reflects the need for decentralization originating from the increase in the number of contributors, the emergence of independent technique services, and the need for quality control for each technique. The reorganized IERS should be in operation by the end of 2000 .

\subsection{IERS Objectives, Products, Observation Techniques and Orga- nizational Components}

According to the IERS Terms of Reference the primary objectives of the IERS are to serve the astronomical, geodetic and geophysical communities by providing the following:

- The International Celestial Reference System (ICRS) and its realization, the International Celestial Reference Frame (ICRF).

- The International Terrestrial Reference System (ITRS) and its realization, the International Terrestrial Reference Frame (ITRF).

- Earth orientation parameters required to study Earth orientation variations and to transform between the ICRF and the ITRF: Polar motion, Universal time, Precession and Nutation.

- Geophysical data to interpret time/space variations in the ICRF, ITRF or Earth orientation parameters, and model such variations.

- Standards, constants and models (i.e., conventions) encouraging international adherence.

IERS collects, archives and distributes products to satisfy the objectives of a wide range of applications, research and experimentation. These products include the following:

- International Celestial Reference Frame.

- International Terrestrial Reference Frame. 
- Monthly Earth orientation data.

- Daily rapid service estimates of near real-time Earth orientation data and their predictions.

- Announcements of the differences between astronomical and civil time for time distribution by radio stations.

- Leap second announcements.

- Products related to global geophysical fluids such as mass and angular momentum distribution.

- Annual report and technical notes on conventions and other topics.

- Long term Earth orientation information.

The accuracies of these products are sufficient to support current scientific and technical objectives including the following:

- Fundamental astronomical and geodetic reference systems.

- Monitoring and modeling Earth rotation/orientation.

- Monitoring and modeling deformations of the solid Earth.

- Monitoring mass variations in the geophysical fluids, including the atmosphere and the hydrosphere.

- Artificial satellite orbit determination.

- Geophysical and atmospheric research, studies of dynamical interactions between geophysical fluids and the solid Earth.

- Space navigation.

The above products are based on the analysis of data observed by the following techniques:

- Very Long Baseline Interferometry (VLBI)

- Lunar Laser Ranging to the Moon (LLR)

- Satellite Laser Ranging (SLR)

- Global Positioning System (GPS)

- Doppler Orbitography System (DORIS)

The current organizational components of the IERS are the following:

- Technique station networks

- Operational and scientific analysis centers 
- Coordination Centers for each technique and for Monitoring Global Geophysical Fluids (MGGF)

- Central Bureau and Sub-bureaus

- Directing Board

As mentioned, this structure will change (see later) to reflect the new Terms of Reference.

\section{Highlights of Activities}

IERS has been a most active organization and hopefully its full history will be written some day. The following examples may serve as indicators of the vitality of the organization:

- The Directing Board met twenty five times between 1988 and 1999. The business conducted has been well-recorded in the published Minutes.

- The IERS Workshops and their Proceedings had a profound impact on the Earth sciences. These included the Workshops in 1990 (Paris); 1991 (Mizusawa); 1992, 1993, 1994 with IGS, 1995 with IGN, 1996 (Paris); 1998 (Potsdam). The 1996 Workshop on IERS Missions, Present and Future (IERS Tech. Note 22) has triggered the 1998 Workshop and Retreat on the reorganization of IERS, now in progress.

- The IERS Standards and Conventions of 1992 and 1996 (IERS Tech. Notes 13 and 21), outgrowths from the MERIT Campaign, and intended primarily for the use of the IERS Analysis Centers, have been now generally accepted by the entire space geodetic community.

In the space available for this article, only the highlights of the full history can be mentioned and even these only in a very selective fashion.

\subsection{Individual Contributions to the IERS}

Individuals make history. To acknowledge the contributions of key individuals in the IERS organization, they are listed below together with their responsibilities.

- Directing Board Chairs: G. Wilkins 1988, K. Yokoyama 1988-1992, Y. Yatskiv 1992-1995, C. Reigber 1995-.

- Directors of the Central Bureau: M. Feissel 1988-1997, D. Gambis 1998-.

- Coordination Centers and Coordinators:

- VLBI: NOAA (W. Carter 1988-1996), NEOS (C. Ma 1996-)

- SLR: University of Texas (B. Schutz 1988-)

- LLR: OCA/CERGA (C. Veillet 1988-1996, P. Shelus 1996-)

- GPS: JPL (B. Melbourne 1989/1992-) 
- DORIS: IGN (P. Willis 1994/1996-)

- MGGF: GSFC/LTP (B. Chao 1998-)

- Central Bureau Sections and Heads:

- Celestial Reference Frame: J.F. Lestrade/ E.F. Arias

- Terrestrial Reference Frame: C. Boucher

- Earth Orientation: D. Gambis

- Sub-Bureaus of the Central Bureau and Heads:

- Atmospheric Angular Momentum: A. Miller/R. Rosen/D. Salstein

- Rapid Service and Predictions: D. McCarthy

\subsection{Progress in Earth Orientation Parameter (EOP) Determination}

The following tables are self-explanatory in describing the progress made in EOP determination. Table 1 shows the development of precision in terms of one daily value of EOP ( $\mathrm{x}, \mathrm{y}$ are the coordinates of the pole, UT1 is Universal Time, and $\mathrm{d} \psi$ and $\mathrm{d} \epsilon$ are corrections to the standard nutation series in longitude and obliquity, respectively).

Table 1. Development of EOP Precision.

\begin{tabular}{lcccccc}
\hline \hline Period & $1962-67$ & $1968-71$ & $1972-79$ & $1980-83$ & $1984-95$ & $1996-$ \\
\hline x (mas) & 30 & 20 & 10 & 2 & 0.7 & 0.2 \\
y (mas) & 30 & 20 & 10 & 2 & 0.7 & 0.2 \\
UT1 (0.1 ms) & 20 & 15 & 10 & 7 & 0.5 & 0.2 \\
d $\psi$ (mas) & 12 & 9 & 5 & 5 & 1.3 & 0.7 \\
d $\epsilon$ (mas) & 2 & 2 & 2 & 2 & 0.6 & 0.3 \\
\hline
\end{tabular}

Table 2 contains the current EOP time resolution and precision figures by observation technique. IERS believes that a factor of 2 improvement is feasible for EOPs.

Fig. 1 indicates the evolution of contribution of each technique in terms of the weights used in the combination solutions. It is easily seen that current UT determination is based only on VLBI, and polar motion mainly on GPS and to lesser extent on SLR and VLBI. The important contribution of SLR is more evident in the determination of the origin of the ITRF with respect to the geocenter.

Finally, Table 3 shows the evolution of operational and analysis centers by technique. The progress is obvious.

\subsection{Progress in the Definition and Maintenance of the ITRF}

Historically the ITRFs have been realized as a set of Cartesian coordinates and velocities of the various contributing space observing sites (e.g., Table 4). The first ITRF, designated ITRF-0, evolved from the BIH-maintained conventional 
Table 2. Current Time Resolution and Precision by Technique.

\begin{tabular}{lcccl}
\hline \hline Technique & $\begin{array}{c}\text { Date } \\
\text { Started }\end{array}$ & $\begin{array}{c}\text { Quantity } \\
\text { Measured }\end{array}$ & $\begin{array}{c}\text { Integration } \\
\text { Time }\end{array}$ & Precision \\
\hline Astrometry & 1899 & Pole & 5 days & $20 \mathrm{mas}$ \\
& & UT1 & $"$ & $1 \mathrm{~ms}$ \\
& & Nutation & $"$ & $40 \mathrm{mas}$ \\
\hline DOPPLER & 1972 & Pole & 2 days & $10 \mathrm{mas}$ \\
\hline LLR & 1969 & UT0 & 1 day & $0.1 \mathrm{~ms}$ \\
\hline SLR & 1976 & Pole & 3 days & $0.3 \mathrm{mas}$ \\
& & LOD & $"$ & $0.2 \mathrm{~ms} / \mathrm{d}$ \\
\hline VLBI & 1981 & Pole & 7 days & $0.3 \mathrm{mas}$ \\
& & Nutation & $"$ UT & $0.15 \mathrm{mas}$ \\
& & UT1 & sub-daily-7 days & $15 \mu \mathrm{s}$ \\
\hline GPS & 1993 & Pole & sub-daily & $0.2 \mathrm{mas}$ \\
& & LOD & " & $0.25 \mathrm{~ms} / \mathrm{d}$ \\
\hline DORIS & 1995 & Pole & 3 days & $1 \mathrm{mas}$ \\
\hline
\end{tabular}

Table 3. Evolution of Contributing Centers to the IERS by Technique.

\begin{tabular}{|c|c|c|}
\hline \multirow{4}{*}{1988} & Operational & Annual Global Analysis \\
\hline & 2 VLBI & 5 VLBI \\
\hline & 1 LLR & 4 LLR \\
\hline & 2 SLR & 5 SLR \\
\hline & Total 5 & 14 \\
\hline \multirow[t]{6}{*}{1998} & 3 VLBI & $7 \mathrm{VLBI}$ \\
\hline & 1 LLR & 4 LLR \\
\hline & $3 \mathrm{SLR}$ & 10 SLR \\
\hline & 7 GPS & 7 GPS \\
\hline & Total 16 & 2 DORIS \\
\hline & & 29 \\
\hline
\end{tabular}

Table 4. Technique Contributions to ITRF97.

\begin{tabular}{cccc}
\hline \hline Technique & No. of Sites & $\begin{array}{c}\text { Position } \\
\text { Uncertainty } \\
(\mathrm{mm} / \mathrm{y})\end{array}$ & $\begin{array}{c}\text { Velocity } \\
\text { Uncertainty } \\
(\mathrm{mm} / \mathrm{y})\end{array}$ \\
\hline VLBI & 129 & 3 & 1 \\
SLR & 129 & 8 & 2 \\
GPS & 231 & 5 & 2 \\
DORIS & 73 & 25 & 8 \\
\hline
\end{tabular}




\section{CONTRIBUTION OF THE TECHNIQUES TO THE IERS COMBINED SOLUTIONS}$$
\text { POLAR MOTION }
$$
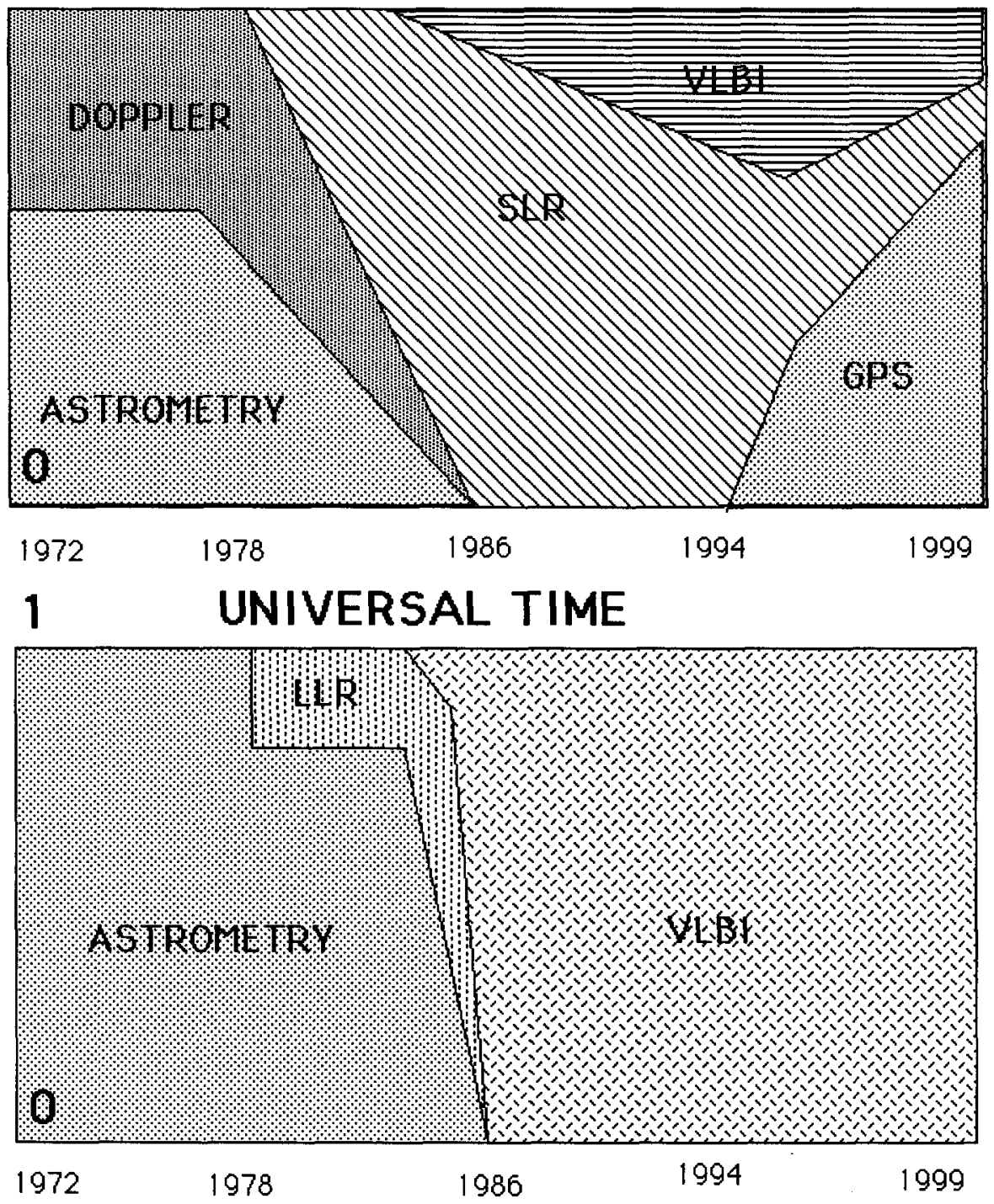

Figure 1. Technique Contribution to IERS Combination Solutions. 
terrestrial reference frame, and dates back to 1989. Since then annual ITRFs have been published (http://lareg.ensg.ign.fr/ITRF).

All techniques can potentially contribute to the ITRF when given appropriate weights and after allowing for systematic differences. However, in the past, certain techniques were given preference (for good reasons) to define e.g., the ITRF origin (SLR) or orientation (VLBI). With the improvement of technology and analysis techniques as well as the proliferation of observing sites, a need for change in procedure was recognized and an IERS Working Group on the ITRF Datum was established in 1998. The report of the Working Group (ftp://hpiers.obspm.fr/iers/itrf/ITRF-WG.Report) sets forth recommendations as to how the ITRF should be established and maintained in the future. Its concept is based on IUGG Resolution No. 2, adopted in 1991 at the 20th General Assembly in Vienna (Appendix, IERS Technical Note 13). Since the recommendations of this report also shed light on current procedure, it is reproduced below in part.

ITRF Origin. To account for geocenter motions, it is necessary to elaborate the expression in Chapter 5, IERS TN 21 for the basic transformation from the ITRF to an Earth-centered inertial frame, ECI, (relatable to the ICRF) as

$$
[E C I]=[P][N][R][W]\{[I T R F]-[O]\}
$$

where $P, N, R$, and $W$ are the usual transformation matrices for precession, nutation, rotation, and polar motion; all of these are time-dependent and computed explicitly with respect to the Earth's center of mass. $O$ is a new time-dependent vector, which gives the translation from the ITRF origin to the instantaneous geocenter, defined to be the center of mass of the Earth including oceans and atmosphere.

The instantaneous vector position of a point on the Earth's surface can be expressed in the ITRF as

$$
X(t)=X_{0}+V_{0}\left(t-t_{0}\right)+\sum \delta X_{i}(t)
$$

where $X(t)$ is the vector position of the point relative to the ITRF origin as a function of time $t ; X_{0}$ and $V_{0}$ are the vector position and velocity of the point at the reference epoch $t_{0}$; the set $\left\{X_{0}, V_{0}\right\}$ is usually regarded as constituting the ITRF realization; $\delta X_{i}(t)$ are site-specific corrections due to various timevarying effects including solid Earth tidal displacements (the full tidal correction including the effect of the permanent tide), ocean tidal loading, etc.;

The origin realized by the ITRF94 frame is adopted as defining the conventional origin of the ITRF. Subsequent ITRF realizations will maintain the ITRF94 origin thereafter by successive optimal Helmert alignments.

ITRF Scale. The ITRF scale should not be considered subject to conventional definition (which would be equivalent to redefining the timescale). Each ITRF realization should inherit the optimally weighted scale (and time evolution) of the input frames that are combined. In practice, it is usually necessary to apply a rescaling of $\left[1+U_{e}\right]$, equivalent to $[1+(0.7 p p b)]$, to all the contributed reference frames in order to comply with the IAU/IUGG recommendations on the use of the TCG timescale. 
Until it is demonstrated that the absolute scale of GPS-based frames is reliable at about the $1 \mathrm{ppb}$ level, these should not be used in the realization of the ITRF scale.

ITRF Orientation. No change is recommended in the current procedure of aligning the axes of each new ITRF realization to remove any net rotational offsets relative to the previous realization.

The IUGG recommends that the ITRF have no global residual rotation with respect to horizontal motions of the Earth's surface. This is achieved by constraining the ITRF so that there are no net rotational rates relative to a global plate motion model, currently the NNR-NUVEL-1A model, with possible recent refinements, is regarded as best for this purpose.

Use of Full Variance-Covariance Matrices. Future ITRF combinations should use only those solutions which are submitted with full variance-covariance information accompanied by the complete a priori constraint matrices. Alignments in origin and orientation of successive ITRF realizations rely on the use of the full covariance matrices.

The Analysis Centers and the technique services are encouraged to develop methods and procedures to include Earth orientation parameters together with their terrestrial frames. This would allow joint simultaneous combination of ITRF and EOP results and should permit greater consistency to be achieved between EOP and ITRF results.

For less demanding users the IERS is considering the establishment of a more traditional terrestrial reference frame which would not be redefined as frequently as the ITRFs are, and would be more accessible in the different geographic areas of the world.

\subsection{Progress in the Definition and Maintenance of the ICRF}

Adopting extragalactic directions as primary celestial references instead of star positions has been envisioned for over a decade by the International Astronomical Union. A number of activities have taken place:

- Working Group on Reference Systems (1988-1991) recommendations dealt with both the fundamental concept space-time references and their actual realizations.

- Working Group on Reference Frames (WGRF) (1991-94) produced a list of about 600 radio sources that could be used to realize an extragalactic celestial reference system.

- The IERS had initiated and developed operational procedures to maintain the celestial reference system.

- The WGRF (1994-97) concluded by recommending that the 1997 IAU General Assembly adopt the IERS celestial reference system under the name International Celestial Reference System (ICRS).

- At its 23rd General Assembly in August 1997 in Kyoto, the IAU decided that, 
- as from 1 January 1998, the IAU celestial reference system shall be the International Celestial Reference System (ICRS) as specified in the 1991 IAU Resolution on Reference Systems and as defined by the International Earth Rotation Service (IERS);

- the corresponding fundamental reference frame shall be the International Celestial Reference Frame (ICRF) constructed by the WGRF;

- the Hipparcos Catalogue shall be the primary realization of the ICRS at optical wavelengths;

- IERS should take appropriate measures, in conjunction with the WGRF, to maintain the ICRF and its ties to the reference frames at other wavelengths.

These activities thus resulted in the fact that as of 1 January 1998, the celestial reference system adopted by the IAU is based on a set of extra galactic directions, replacing the Fundamental Catalog No. 5 (FK5), based primarily on the dynamics of the solar system. This implies a drastic modification in the underlying concepts and has practical consequences at various levels for the users needing the best astrometric accuracy ( 50 mas). The IAU decision began a new era in the definition of reference systems and marked a sharp break with the practice of the last three hundred years characterized by the long series of fundamental star catalogues.

The origin of the ICRS axes is located at the barycentre of the solar system and the directions of its axes are fixed relative to distant extragalactic sources. The origin of right ascensions is also defined consistently with that of the FK5 by fixing the right ascension of $3 \mathrm{C} 273 \mathrm{~B}$ to the $\mathrm{FK} 4$ value transformed to the $\mathrm{J} 2000$ (FK5) System. For the sake of continuity with the FK5 pole, the ICRS pole is in the J2000.0 direction defined by the conventional IAU models for precession and nutation.

The ICRF consists of a catalogue of equatorial coordinates of 608 extra galactic radio sources derived from about 1.6 million observations accumulated by a worldwide VLBI network over 1979-1995. It includes three lists of objects, with the

- most compact and best observed 212 defining sources, with a median uncertainty of 0.4 mas on individual positions,

- compact sources (294) whose positions are likely to be improved when more observations are accumulated in the future,

- sources less appropriate for astrometric purpose (102), but which are provided for possible ties of reference frames at other wavelengths or for other objectives.

A first extension of ICRF, ICRF-Ext.1, is now available, and the data analysis is described in the 1998 IERS Annual Report. The primary objectives of this first maintenance and extension of the ICRF are to provide positions for extragalactic radio sources observed since July 1995 and to refine the positions of candidate sources from additional observations. The secondary objectives include monitoring sources to ascertain whether they continue to be suitable for 
their use in the ICRF and improving the data analysis. The catalog ICRF-Ext. 1 contains all the 667 observed extragalactic sources in the VLBI astrometric/geodetic data set through April 1999. It is available on ftp and web at the following address:

Web: http://hpiers.obspm.fr

under "results: The International Celestial Reference Frame (ICRF)"

(Web file: webiers/results/icrf/Icrf.html)

To appreciate the progress made, compare the above to the first ICRF-0, which in 1989 contained 228 sources including 28 defining ones.

For all users with accuracy requirement less stringent than 50 mas, the adoption of ICRS/ICRF has no significant effect.

\subsection{Progress in MGGF Investigations}

As indicated in the Abstract, progress in IERS efforts in global geophysical fluid monitoring and in "geocenter" monitoring is not the subject of this paper. The results of such investigations are reported elsewhere in this volume and in the IERS Technical Note 25, 26, and 28.

\section{Future Development}

As mentioned, the IERS is being restructured as per the twenty-six recommendations made at the 1996 Paris Workshop, followed be the decisions at the 1998 Potsdam Retreat. The new Terms of Reference (see http://hpiers .obspm.fr) adopted by the Directing Board in 1999, call for a more decentralized structure illustrated in Fig. 2, presented at the 1999 IUGG General Assembly in Birmingham by the Chair of the Directing Board.

In the new structure the responsibility for quality control shifts from the Central Bureau to the Technique Centers and for combination solutions to the Analysis Coordinator. In addition, the Product Centers are responsible for the various IERS products.

There are other observational techniques and analysis related developments, which may have profound effects on the work of the IERS. These include the following:

- Development of new transportable VLBI systems with fiber optic communication, and space VLBI missions.

- Development of the SLR2000 system with reduced data latancy.

- Improvements in GPS/GLONASS receiver technology and satellite availability.

- Use of the forthcoming Low Earth Orbiting (LEO) satellites.

- Development of the GALILEO system: 20-30 satellite configuration, transmitting on GPS/GLONASS frequencies and equipped with a 2-way ranging system. 


\section{New Structure International Earth Rotation Service}

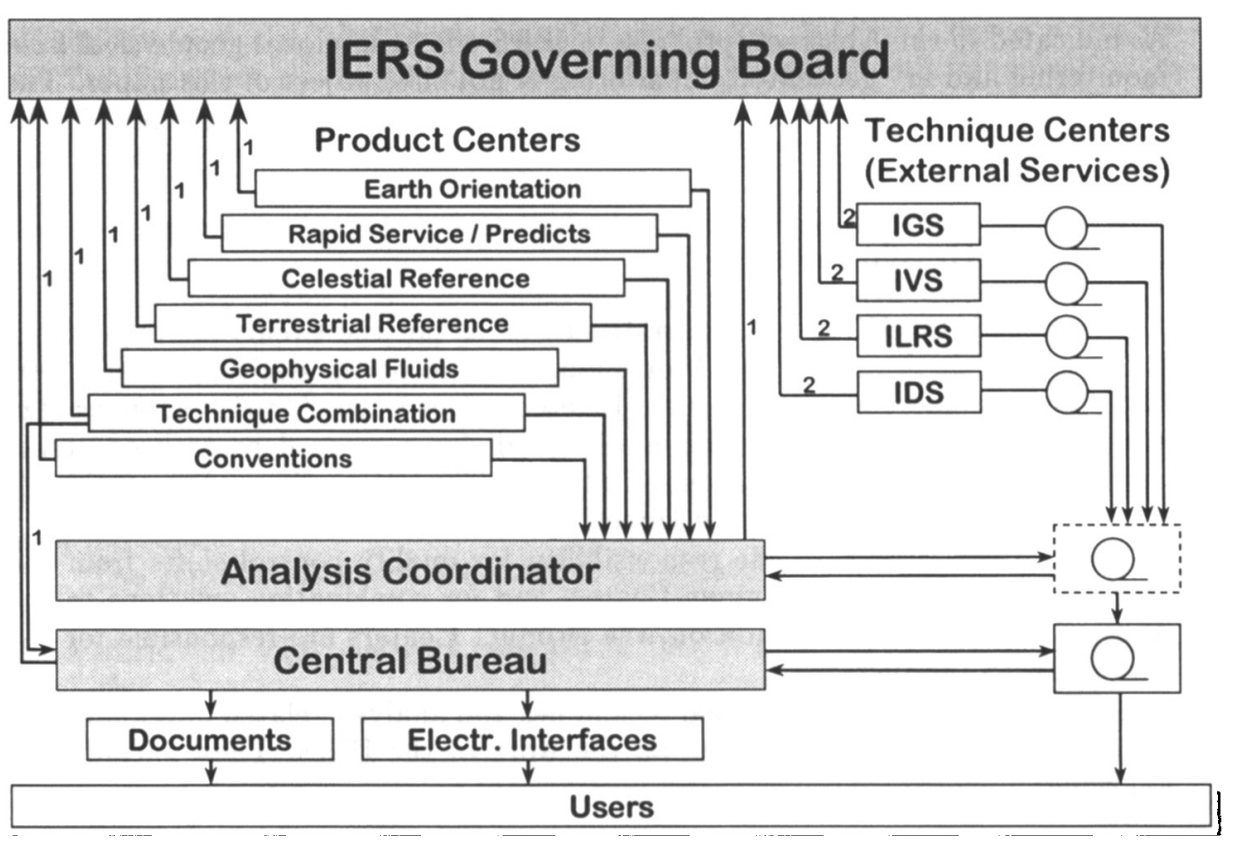

Figure 2. New Structure of the IERS. 
- Improved gravity modeling by observing the forthcoming gravitational satellites (e.g., CHAMP, GRACE).

- Improved ITRF to the mm level.

These developments may lead to a factor of $2-3$ improvements in the EOP products during the next decade.

\section{Epilogue}

The author's relationship with the IERS has been always friendly, unofficial and somewhat ambiguous. Therefore it was somewhat of a surprise when the organizers of this symposium requested him to present the history of the IERS during the last decade, instead of someone officially engaged in the IERS. Nevertheless it is hoped that the above attempt, incomplete as it is, allows the reader a fair and representative glimpse of the remarkable accomplishments of this organization and the hard work of the persons associated with it.

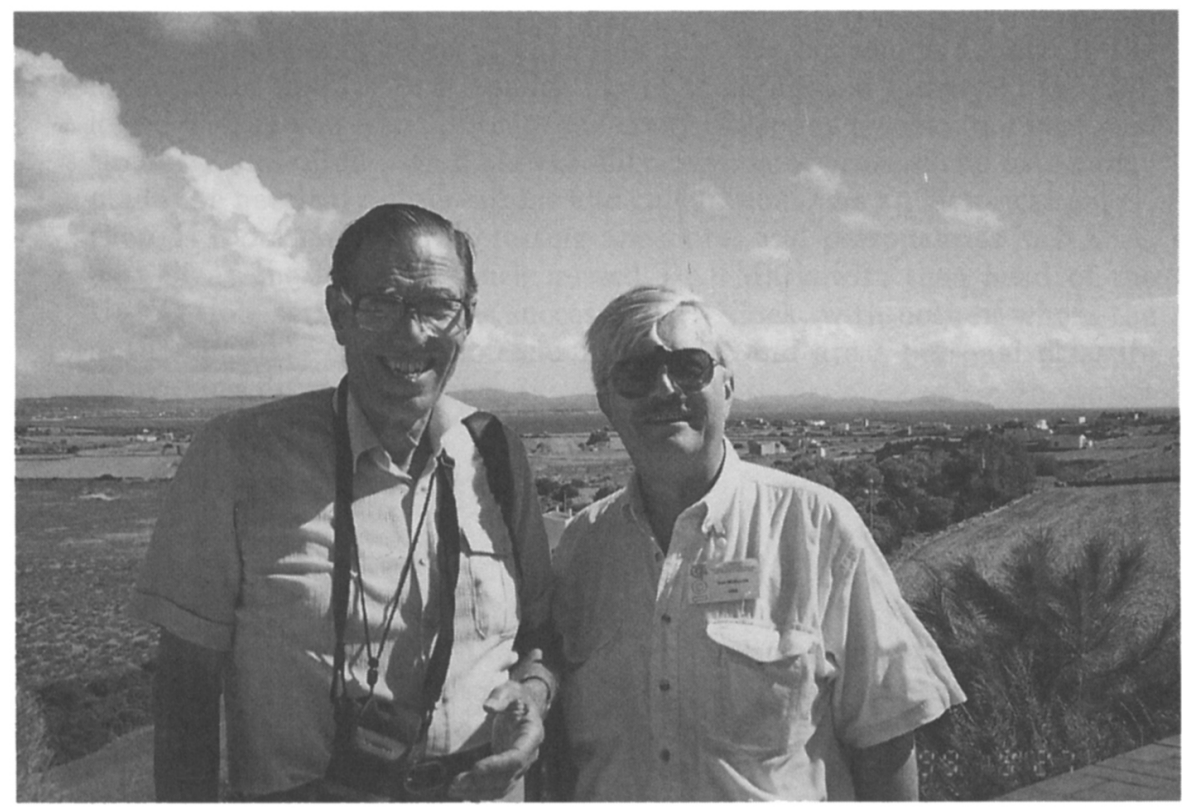

George Wilkins and Ivan Mueller 